\title{
Fermentation and dry fractionation increase bioactivity of cloudberry (Rubus chamaemorus)
}

\section{Puupponen-Pimiä, Riitta}

2015-11-14

Puupponen-Pimiä , R , Nohynek , L , Juvonen , R , Kössö , T , Truchado , P , Westerlund-Wikström , B A , Leppänen , T , Moilanen , E \& Oksman-Caldentey , K-M 2015 ,

' Fermentation and dry fractionation increase bioactivity of cloudberry (Rubus chamaemorus)

pÿ' , Food Chemistry , vol. 197 , no. Part A , pp. 950958 . https://doi.org/10.1016/j.foodchem.2015.11.061

http://hdl.handle.net/10138/324011

https://doi.org/10.1016/j.foodchem.2015.11.061

publishedVersion

Downloaded from Helda, University of Helsinki institutional repository.

This is an electronic reprint of the original article.

This reprint may differ from the original in pagination and typographic detail.

Please cite the original version. 


\title{
Fermentation and dry fractionation increase bioactivity of cloudberry (Rubus chamaemorus)
}

\author{
Riitta Puupponen-Pimiä ${ }^{a, *}$, Liisa Nohynek ${ }^{a}$, Riikka Juvonen ${ }^{a}$, Tuija Kössö ${ }^{a}$, Pilar Truchado ${ }^{a}$, \\ Benita Westerlund-Wikström ${ }^{\mathrm{b}}$, Tiina Leppänen ${ }^{\mathrm{c}}$, Eeva Moilanen ${ }^{\mathrm{c}}$, Kirsi-Marja Oksman-Caldentey ${ }^{\mathrm{a}}$ \\ ${ }^{a}$ VTT Technical Research Centre of Finland Ltd., P.O. Box 1000, FI-02044 VTT, Finland \\ ${ }^{\mathrm{b}}$ Division of General Microbiology, Department of Biosciences, University of Helsinki, P.O. Box 56, FI-00014 University of Helsinki, Helsinki, Finland

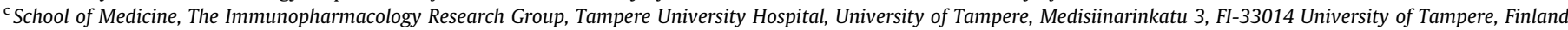

\section{A R T I C L E I N F O}

\section{Article history:}

Received 23 June 2015

Received in revised form 3 November 2015

Accepted 13 November 2015

Available online 14 November 2015

\section{Keywords:}

Cloudberry

Bioprocessing

Fermentation

Dry fractionation

Antimicrobial activity

Antiadhesion activity

Anti-inflammatory activity

Phenolic compounds

\begin{abstract}
A B S T R A C T
Phenolic composition and bioactivity of cloudberry was modified by bioprocessing, and highly bioactive fractions were produced by dry fractionation of the press cake. During fermentation polymeric ellagitannins were partly degraded into ellagic acid derivatives. Phenolic compounds were differentially distributed in seed coarse and fine fractions after dry fractionation process. Tannins concentrated in fine fraction, and flavonol derivatives were mainly found in coarse fraction. Ellagic acid derivatives were equally distributed between the dry fractions. Fermentation and dry fractionation increased statistically significantly anti-adhesion and anti-inflammatory activity of cloudberry. The seed fine fraction showed significant inhibition of P fimbria-mediated haemagglutination assay of uropathogenic Escherichia coli. The seed coarse fraction significantly reduced NO and IL-6 production and iNOS expression in activated macrophages. Fermentation did not affect antimicrobial activity, but slight increase in activity was detected in dry fractions. The results indicate the potential of cloudberry in pharma or health food applications.
\end{abstract}

(c) 2015 Elsevier Ltd. All rights reserved.

\section{Introduction}

Fermentation technologies with microbes are widely used in the processing of plant-based materials, in order to enhance selflife, texture, flavour and bioactivity of food and feed products. Starter technology, in which the berries are inoculated with wellcharacterised microbes, is thus a promising tool to modify phenolic compounds and thus enhance the bioactivity of berry products. We recently developed fermentation technology for lingonberry, in which fermentation of lingonberry with Hanseniaspora uvarum yeast increased the contents of the natural preservative, benzoic acid (Viljanen, Heiniö, Juvonen, Kössö, \& Puupponen-Pimiä, 2014).

Traditional jams and juices are still the main products of the berry processing industry. Due to the seeds in Rubus berries, such as cloudberry, being rather large, the food industry often separates the juice from press cake and uses only juice for further products. The remaining press cake, which contains mainly seeds and peel, can be used to extract valuable seed oil. However, still today in many cases berry by-products have no application, although they

\footnotetext{
* Corresponding author.

E-mail address: Riitta.puupponen-pimia@vtt.fi (R. Puupponen-Pimiä).
}

are very rich in bioactive compounds, and they may cause environmental and storage problems. Thus, new innovative applications for berry by-products are needed by the industry. Enzymeassisted berry processing, using microbially-based pectinolytic enzymes, has been studied by Puupponen-Pimiä, Nohynek, Ammann, Oksman-Caldentey, and Buchert (2008). In that study, commercial pectinase preparations released phenolic compounds from the cell wall matrix and consequently enhanced antimicrobial and antioxidant activity of the berry juice and press cake significantly.

Cloudberry (Rubus chamaemorus) is a unique Nordic berry species. In particular, the northernmost region, Lapland, is a favourable growth zone for this berry. The plant produces ambercoloured, juicy, flavourful and fragrant berries, which are an important raw material for the food, beverage and cosmetic industries. Cloudberry is very rich in ellagic acid, which is present in the plants mainly in the form of polymeric ellagitannins (ETs) which are esters of glucose with ellagic acid. More detailed analyses of Kähkönen, Kylli, Ollilainen, Salminen, and Heinonen (2012) revealed the presence of altogether 26 ellagitannin compounds in the cloudberry fruit, with lambertianin C (2804 Da) and sanguiin H-6 (1870 Da) being the two major components. 
Recent studies have shown that ellagic acid and ETs possess many interesting biological activities and thus they may play preventive role in disease prevention. For example cardioprotective effects of ellagitannins have been recently discussed (Larrosa, García-Conesa, Espín, \& Tomás-Barberán, 2010). ETs have also shown beneficial effects on brain function: Rojanathammanee, Puig, and Combs (2013) reported potential attenuation in Alzheimeŕs disease progression due to anti-inflammatory effects in brains caused by ETs. In a recent study Sangiovanni et al. (2013) showed for the first time the preventative effect of ETs in gastric inflammation. Our clinical study indicated that bioavailability of ETs appears to be dependent on the composition of gut microbiota (Puupponen-Pimiä et al., 2013). In our in vitro studies, berry ETs showed strong antimicrobial activity against many severe human pathogens and spoilage bacteria, suggesting their potential role as natural antimicrobials and preservatives (reviewed by Puupponen-Pimiä, Nohynek, Alakomi, and Oksman-Caldentey (2005)). Adhesion to host cells is an important initiating step in bacterial infections. A number of reports indicates that berry phenolic compounds can inhibit bacterial adhesion to human cells in vitro (reviewed by Signoretto, Canepari, Stauder, Vezzulli, and Pruzzo (2012)), but to our knowledge the antiadhesive effect of cloudberry phenolics has so far not been investigated.

The aim of this study was to investigate how bioprocessing with food-grade lactic acid bacteria and further mechanical fractionation of the press cake affect composition of cloudberry phenolics and bioactivity of the cloudberry. Special interest was directed to antimicrobial, anti-adhesion and anti-inflammatory activities. We also developed an innovative dry fractionation method for the berry press cake to produce ingredients high in phenolic phytochemicals suitable for various industrial applications.

\section{Materials and methods}

\subsection{Berry material and pre-treatments}

Frozen, ripe cloudberries ( $R$. chamaemorus) of Finnish origin were obtained from a local distributor, Pakkasmarja Ltd. (Suonenjoki, Finland). The cloudberries were frozen and stored at $-20^{\circ} \mathrm{C}$ until use.

Before the fermentation berries were heat-treated in order to guarantee microbiological safety and uniform quality of the fermentation product. Frozen cloudberries and ultra-pure water were mixed together $(1: 1)$. The mixture was heated in a water bath to $80^{\circ} \mathrm{C}$ and held there for $5 \mathrm{~min}$. After that the mixture was cooled in an ice bath and berries were crushed by a sterile potato masher. The $\mathrm{pH}$ of the mixture was adjusted to 5.0 with $5 \mathrm{~N}$ sterile filtered sodium hydroxide.

\subsection{LAB fermentation of cloudberries}

Processing steps of cloudberry are shown in Fig. 1. Pediococcus pentosaceus VTT E-072742 from VTT Culture Collection (http://culturecollection.vtt.fi/) was used as a starter culture in fermentation of cloudberries. This strain was chosen due to its ability to grow in the cloudberry material. Prior to fermentations, the strain was refreshed in De Man Rogosa Sharpe broth (MRS; Oxoid, Basingstoke, UK) for 1 day in a 100\% carbon dioxide atmosphere which was created using anaerobic jars and Anaerocult C strips (Merck, Darmstadt, Germany). The cells were collected from refreshed cultures by centrifugation and washed once in Ringeís solution (Merck, Darmstadt, Germany). The fermentations were carried out as recently described by Viljanen et al. (2014). Briefly, the heattreated crushed berry material was inoculated with approximately $10^{6} \mathrm{cfu} \mathrm{g}^{-1}$ of washed bacterial cells. The fermentations were per- formed on a $6-\mathrm{kg}$ scale in a $15-\mathrm{L}$ capacity bioreactor for 14 days at $30^{\circ} \mathrm{C}$ under constant mixing $(130 \mathrm{rpm})$. The bioreactor was purged with sterile-filtered nitrogen gas in order to create anaerobic conditions. The viable counts of lactic acid bacteria and yeasts were determined before and after the fermentations using plate count technique as previously described (Katina et al., 2007). The results were expressed as colony-forming units (CFU) per gram of wet weight (ww). The $\mathrm{pH}$ value was measured with a standard $\mathrm{pH}$ meter. For chemical analysis samples of the fermented materials were stored frozen until analysed.

\subsection{Juice pressing and dry fractionation of the press cake}

After fermentation the berry mash was treated with a hydraulically operated high-pressure tincture press (HAFICO, Fischer and Co., Dusseldorf, Germany) using 5 litres filling material to separate juice and insoluble press cake, which was further processed.

The press cake was dried in a fluid bed dryer (model TG 200, Retsch, Haan, Germany) using $+45^{\circ} \mathrm{C}$ air flow, and a dry fractionation method was then developed and applied. The press cake was dry fractionated by sieving using different sieve sizes and by milling in a disc mill into pulp, peel, seed, seed fine and seed coarse fractions. Mass yields of different fractions were determined. Colour and surface features of the samples were examined with Zeiss SteREO Discovery.V8 stereomicroscope and imaged using an Olympus DP-25 single chip colour CCD camera and the Cell^P imaging software.

\subsection{Chemical analysis of fermented cloudberries}

The concentration of sugars (glucose, fructose and sucrose) were analysed by high-performance anion exchange chromatography (HPAEC) (Dionex ICS-3000) with pulse amperometric detection (PAD) (Dionex Corporation, Sunnyvale, CA) as described by Viljanen et al. (2014). The separation of organic acids and mannitol was carried out on an Aminex HPX-87H column $(300 \times 7.8 \mathrm{~mm}$; BioRad, Hercules, CA) as described by Viljanen et al. (2014).

\subsection{Preparation and analysis of phenolic extracts}

Concentrated phenolic extracts were prepared from cloudberry fermentation samples before juice pressing (days 0, 7 and 14) and from dry fractions of cloudberry press cake (fine and coarse fractions). Frozen berry samples were first lyophilised and ground to a fine powder, and then extracted according to Kähkönen, Hopia, and Heinonen (2001) with slight modifications. Phenolic compounds were extracted from $10 \mathrm{~g}$ of dry berry samples with $20 \mathrm{~mL}$ of solvent (acetone:water 70:30 vol/vol) in centrifuge tubes. Tubes were centrifuged ( $3000 \mathrm{rpm}, 5 \mathrm{~min}$ ), and the clear supernatant was collected. The procedure was repeated twice with $10 \mathrm{~mL}$ of solvent. Supernatants were combined and acetone was evaporated. Acidic Milli Q water (0.5\% trifluoroacetic acid) was added to the residue, and interfering sugars and organic acids were removed by solid-phase extraction using C18 columns (Sep-Pak Vac $20 \mathrm{cc}, 5 \mathrm{~g}$; Waters). Phenolic compounds were eluted with methanol. These purified extracts were freeze-dried and stored at $-20^{\circ} \mathrm{C}$ prior to analyses.

Phenolic compounds were analysed from the acetone extracts by HPLC-DAD and selected samples by UPLC-QToF-MS/MS. Analytical HPLC method was modified from methods described by Aaby, Ekeberg, and Skrede (2007), and Määttä-Riihinen, Kamal-Eldin, and Törrönen (2004). The HPLC system consisted of a Waters 600S system controller pump, Waters 717plus autosampler and Waters 2996 series photodiode array detector (Waters, Milford, MA). All the data was processed by Waters Empower Pro chromatography software. Phenolic compounds were separated on a Hypersil BDC 


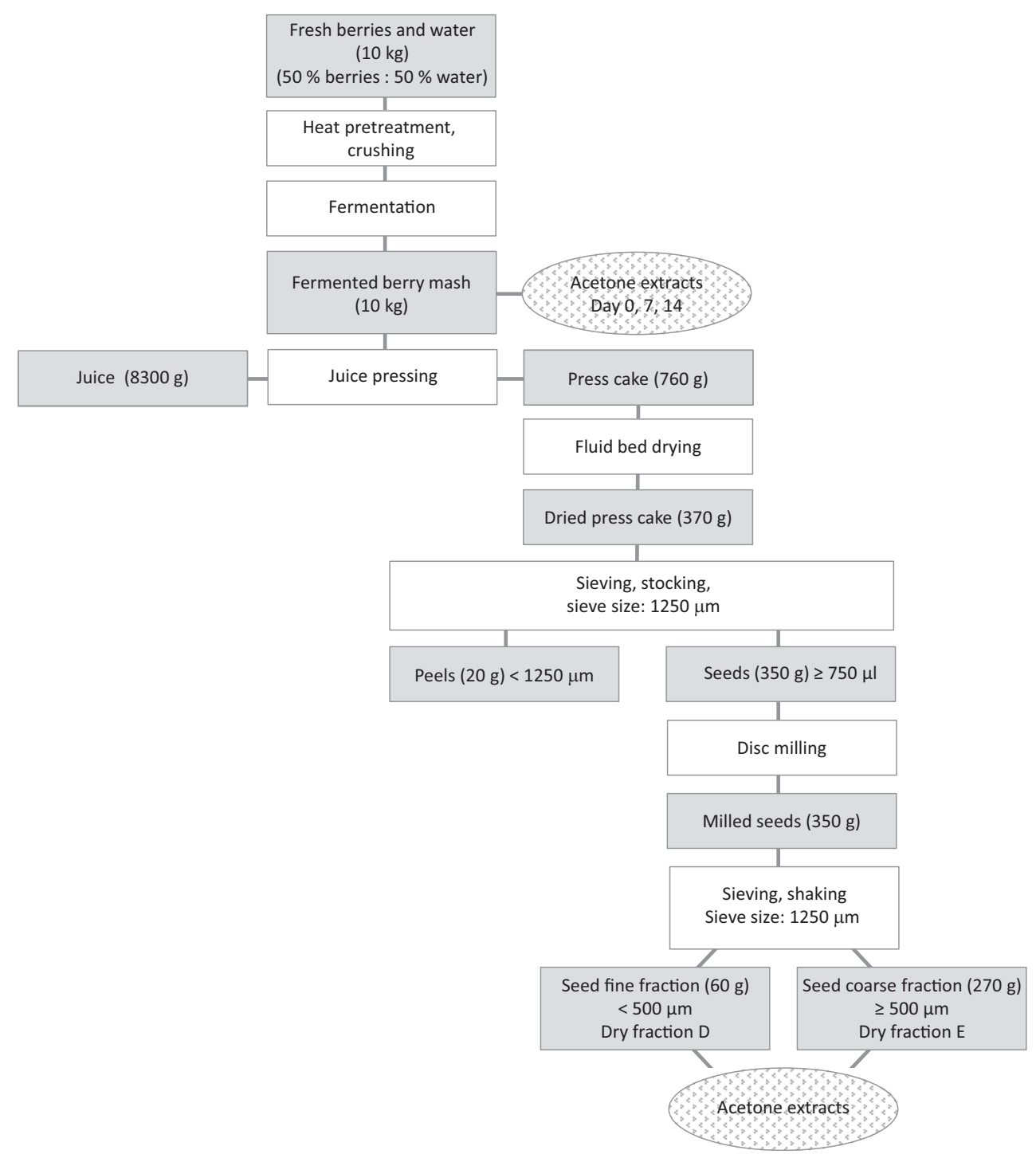

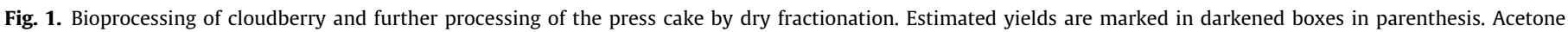
extracts, marked with circles, were prepared for bioactivity testing.

C-18 (150 $\times 4.6 \mathrm{~mm}, 5 \mu \mathrm{m}$, Agilent, Santa Clara, CA) reverse phase column. The solvents used for analyses of flavonols, phenolic acids and ellagic acid derivatives in the gradient program were $5 \%$ formic acid in water (A) and 100\% acetonitrile (B) (Rathburn Chemicals Ltd, Walkerburn, UK) starting with $10 \% \mathbf{B}$ and using a gradient to obtain $25 \%$ at $8 \mathrm{~min}, 65 \%$ at $45 \mathrm{~min}$ and $95 \%$ at $50 \mathrm{~min}$. The solvents used in ellagitannin analyses in the gradient program were $2 \%$ acetic acid in water and acetic acid:acetonitrile:water (2:50:48). The flow rate was $1 \mathrm{~mL} / \mathrm{min}$ and the injection volume $20 \mu \mathrm{l}$. Spectral data from all peaks were accumulated in the range $240-600 \mathrm{~nm}$ and chromatograms were recorded at 280 and $360 \mathrm{~nm}$. The phenolic compounds were quantified according to their UV spectra, and retention time, when possible, with commercially available standards. Ellagitannins were quantified using punicalagin as standard (USA, California). All these standards were purchased from Sigma (St. Louis MO) and punicalagin from Chromadex (USA, California).

The UPLC-MS system consisted of an Acquity UPLC instrument (Waters, Milford, MA) using an Acquity UPLC BEH C18 column $(1.7 \mu \mathrm{m}, \quad 100 \mathrm{~mm} \times 2.1 \mathrm{~mm}$ i.d.) equipped with an Acquity Vanguard pre-column. The column was kept at $35^{\circ} \mathrm{C}$ and the flow rate was $0.3 \mathrm{~mL} / \mathrm{min}$. The mobile phase was eluent $\mathbf{A} 0.1 \%$ formic acid in water:acetonitrile (95:5, v/v) and eluent B, $0.1 \%$ formic acid in acetonitrile. The gradient program was following $0 \mathrm{~min} 0 \% \mathbf{B}$, $22 \min 28 \%$ B, $22.5 \min 40 \%$ B, 23 min $100 \%$ B, $24.5 \min 100 \%$ B, $25 \mathrm{~min} 0 \% \mathbf{B}$. The sample volume injected was $2-5 \mu \mathrm{l}$. The UPLC was connected to a Waters Micromass Qtof Premier Mass Spectrometer (Waters, Milford, MA). An electrospray interface was used in the ionisation, operating both in negative and positive modes and the scanned data was collected in $\mathrm{m} / \mathrm{z}$ range of $100-2000 \mathrm{Da}$. The ionisation source parameters were capillary voltage $3.0 \mathrm{kV}$, cone voltage $30 \mathrm{eV}$, source temperature $120^{\circ} \mathrm{C}$ and collision energies UPLC-MS $3 \mathrm{eV}$ and UPLC-MS/MS $20 \mathrm{eV}$. Desolvation gas temperature was $270{ }^{\circ} \mathrm{C}$ and gas flow-rate $795 \mathrm{~L} / \mathrm{h}$. Nitrogen and argon were used as cone and collision gases, respectively. The MS was calibrated using sodium formate and the lock masses were leucine enkephalin (negative mode) and reserpine (positive mode). Standard mixtures were run both in positive and negative modes and in UPLC-MS and UPLC-MS/MS modes, to help the identification of unknown compounds in the berry extracts. Masslynx software version 4.1 was used in controlling the instruments and in data handling. 


\subsection{Bioactivities}

\subsubsection{Antimicrobial activity}

Antimicrobial activity of the acetone extracts $(1 \mathrm{mg} / \mathrm{mL})$ of the cloudberry material was measured in liquid cultures according to Nohynek et al. (2006). Microbial strains used in the analysis and their origin are presented in Supplementary Material 1. Bacterial strains Staphylococcus aureus VTT E-70045, Pseudomonas aeruginosa VTT E-84219 and Escherichia coli strains VTT E-94564 ${ }^{\mathrm{T}}$ and VTT E-093121 were cultured aerobically at $37^{\circ} \mathrm{C}$ on nutrient agar (NA, Oxoid) or in nutrient broth (NB, Oxoid) with agitation (150 rpm). Lactobacillus rhamnosus GG VTT E-96666 was grown in/on MRS (De Man Rogosa Sharpe; Oxoid, Basingstoke, UK) medium at $37^{\circ} \mathrm{C}$ in an anaerobic chamber. Yeast strains Candida albicans VTT C-85161 and Saccharomyces cerevisiae VTT C-00360 were grown on yeast mould agar (YMA, Difco) or in yeast mould broth (YMB, Difco) with agitation (100 rpm) at $37^{\circ} \mathrm{C}$ and $30^{\circ} \mathrm{C}$, respectively. Microbial stock cultures were maintained frozen at $-80^{\circ} \mathrm{C}$. For experimental use the microbial cultures were transferred onto solid media and incubated for 1-2 days as described above for each strain. The colonies were introduced into liquid media, incubated for $12-24 \mathrm{~h}$, and used as the source of inoculum for antimicrobial activity analysis. The liquid culture method used (Nohynek et al., 2006) was modified by decreasing original $10 \mathrm{~mL}$ culture volume to $0.5 \mathrm{~mL}$, enabling the analysis of very small amounts of berry extracts. Briefly, growth media were inoculated with $0.1 \%$ of overnight microbial cultures and delivered in portions of $0.5 \mathrm{~mL}$ on each berry extract weighed beforehand in round bottom Eppendorf tubes of $2 \mathrm{~mL}$. Cultures with no berry extract were used as positive controls, and cultures with antibiotic compound (chloramphenicol or hygromycin B) were used as negative controls for microbial growth. The cultures in Eppendorf tubes were incubated in their optimal growth conditions, and microbial growth was followed by taking samples of $30 \mu \mathrm{L}$ from the cultures altogether 4 times during the incubation period of $24 \mathrm{~h}$. The samples were diluted in peptone saline (Maximal Recovery Diluent; Lab M, Heywood, UK), and the proper dilutions were plated. The plates were incubated as described above, and, on the basis of the microbial colony counts recorded, the growth curves were described for each culture. The inhibitory effects of berry extracts on the microbes were evaluated by comparing the control growth curves with those obtained from cultures with extracts.

\subsubsection{Anti-adhesion activity analysed by haemagglutination assay} (HA)

Gram-negative bacteria frequently express proteinaceous appendages called fimbriae, which mediate bacterial adhesion to specific receptor molecules on host cells. The non-fimbriated strain E. coli HB101 and the recombinant strain E. coli HB101 (pPIL29115), which expresses $P$ fimbria as the sole fimbria type, were available from previous work (Boyer \& Roulland-Dussoix, 1969; Kylli et al., 2010; van Die, van Megen, Hoekstra, \& Bergmans, 1984). The strains were grown for $18 \mathrm{~h}$ at $37{ }^{\circ} \mathrm{C}$ on Luria agar plates supplemented with appropriate antibiotics. Bacteria were collected and gently suspended in phosphate-buffered saline, pH 7.1 (PBS). The freeze-dried phenolic extracts of cloudberry materials were dissolved at a concentration of $1 \mathrm{mg}$ lyophilised powder per $\mathrm{mL}$ PBS. EDTA-containing human erythrocytes of blood group $\mathrm{O}$ (Ortho-Clinical Diagnostics Inc., Raritan NJ) were washed three times in cold PBS and suspended to $4 \%(\mathrm{v} / \mathrm{v})$ in cold PBS, which resulted in $4-8 \times 10^{8}$ erythrocytes $/ \mathrm{mL}$. The cells were kept at $+4{ }^{\circ} \mathrm{C}$ or on ice.

HA was performed in 96-well round-bottomed microtitre plates as described before (Kylli et al., 2010). Briefly, $50 \mu \mathrm{L}$ of bacterial cells were mixed with $50 \mu \mathrm{L}$ of $4 \%$ erythrocyte suspension; the plates were incubated for $18 \mathrm{~h}$ at $+4{ }^{\circ} \mathrm{C}$ and the HA was read by eye. For inhibition of HA, bacterial cell suspensions were mixed with phenolic extracts, at final concentrations of $0,1.56,3.13$, $6.25,12.5,25,50$ and $100 \mu$ g extract $/ \mathrm{mL}$ suspension $(\mathrm{w} / \mathrm{v})$, prior to the addition of erythrocytes. The optimal concentration of bacterial cells in inhibition of HA was for each analysis determined by two-fold titration of the bacterial suspensions. The concentration used in inhibition of HA was four times the minimum agglutinating concentration, which equalled $2 \times 10^{8}$ to $2 \times 10^{9}$ bacteria/mL. The inhibition analysis was repeated four times for each combination of bacterial strain and phenolic extract. The average minimum inhibitory concentration (MIC; $\mu \mathrm{g}$ extract $/ \mathrm{mL}$ ) and standard deviation were calculated. On the basis of phenolics concentrations determined by analytical HPLC (Table 2), the MICS of ellagitannin, hydroxycinnamic acid, flavonols, ellagic acid and derivatives, and benzoic acid were calculated.

\subsubsection{Anti-inflammatory testing}

Anti-inflammatory activity was studied using murine J774 macrophages (American Type Culture Collection, Rockville, MD), which were cultured at $37{ }^{\circ} \mathrm{C}$ in $5 \% \mathrm{CO}_{2}$ atmosphere in Dulbecco's modified Eagle's medium with Ultraglutamine 1 (Lonza Verviers SPRL, Verviers, Belgium) supplemented with $10 \%$ heat-inactivated foetal bovine serum (Lonza), $100 \mathrm{U} / \mathrm{mL}$ penicillin, $100 \mu \mathrm{g} / \mathrm{mL}$ streptomycin and $250 \mathrm{ng} / \mathrm{mL}$ amphotericin B and harvested with trypsin-EDTA (Invitrogen, Paisley, UK).

Cells were seeded on 6 or 24-well plates and grown for $72 \mathrm{~h}$ to confluence prior to the experiments. Cloudberry acetone extracts were first dissolved in DMSO, and the stock solution was diluted in 1:1000 in fresh culture medium to obtain the final concentrations. Macrophages were activated through TLR4 pathway by adding bacterial lipopolysaccharide (LPS, $10 \mathrm{ng} / \mathrm{mL}$ ) into the culture.

Possible cytotoxicity of the tested extracts was ruled out by measuring cell viability using Cell Proliferation Kit II (a modification of XTT test; Roche Diagnostics, Mannheim, Germany) according to manufacturer's instructions.

The effects of the tested extracts on nitric oxide (NO) production in activated macrophages was determined by measuring the accumulation of nitrite, a stable metabolite of NO, in the culture medium by the method of Griess (Green et al., 1982).

Interleukin-6 (IL-6) concentrations in the culture medium were measured by enzyme-linked immunosorbent assay (ELISA) using reagents from R\&D Systems Europe Ltd (Abingdon, UK).

The effects of the tested extracts on the expression of inducible nitric oxide synthase (iNOS) were determined by Western blotting. Actin was used as a loading control. Protein samples $(20 \mu \mathrm{g}$ of lysates) were analysed according to standard Western blotting protocol as described previously (Leppänen, Jalonen, Korhonen, Tuominen, \& Moilanen, 2010). The membrane was incubated with the primary antibody in the blocking solution at $4{ }^{\circ} \mathrm{C}$ overnight, and with the secondary antibody in the blocking solution for $1 \mathrm{~h}$ at room temperature. Bound antibody was detected using Super Signal ${ }^{\circledR}$ West Pico or Dura chemiluminescent substrate (Pierce, Rockford, IL) and Image Quant LAS 4000 mini imaging system (GE Healthcare Bio-Sciences AB, Uppsala, Sweden). The quantitation of the chemiluminescent signal was carried out with the use of Image Quant TL software (GE Healthcare). Rabbit polyclonal iNOS and actin antibodies and HRP-conjugated goat polyclonal anti-rabbit antibody were from Santa Cruz Biotechnology Inc. (Santa Cruz, CA).

Results were expressed as mean \pm standard error of mean (S.E. M.). Statistical significance of the results was calculated by analysis of variance supported by Dunnett's adjusted significance levels. Differences were considered significant at $p<0.05$. 
Table 1

Organic acids and sugars (mg/g of dry weight) in cloudberry fermentations with $P$. pentosaceus E-072742.

\begin{tabular}{clllll}
\hline Time (days) & $\mathrm{pH}$ & Glucose & Fructose & Lactic acid & Malic acid \\
\hline 0 & 5.1 & 158 & 94 & 0 & 54 \\
7 & 4.4 & 139 & 79 & 59 & 22 \\
14 & 4.1 & 124 & 62 & 97 & 23 \\
\hline
\end{tabular}

\section{Results and discussion}

\subsection{Viable counts and sugar metabolism}

P. pentosaceus VTT E-072742 grew from $2 \times 10^{6} \mathrm{cfu} \mathrm{g}^{-1}$ to $1 \times 10^{8}$ cfu $^{-1}$ during the 7 -day fermentation. The viable counts stayed at this level until the end of the fermentation at day 14 . The growth of contaminating yeast or bacteria was not detected $\left(<10^{2} \mathrm{cfu} / \mathrm{g}\right)$. The cloudberry material acidified during the lactic fermentation. The $\mathrm{pH}$ value decreased from an initial value of 5.1 to 4.1 during the 14 days. The sugar analysis showed that the concentration of glucose and fructose decreased and the concentration of lactic acid increased (Table 1). The concentrations of citric and acetic acids were below the detection limit. The concentration of malic acid decreased during the fermentation, probably due to malolactic fermentation. In malolactic fermentation malic acid is decarboxylated to lactic acid and carbon dioxide, which leads to cytoplasmic alkalinisation and ATP synthesis. Malolactic fermentation has been identified as a major system for alkali production in oral streptococci and for protection against environmental stresses such as acid damage (Sheng, Baldeck, Nguyen, Quivey, \& Marquis, 2010).

\subsection{Juice and press cake dry fractions}

After fermentation the mass yields of cloudberry juice were $81-$ $85 \%$ and yields of press cake were $8-10 \%$. About $5 \%$ of the press cake consisted of peel and pulp, and the remaining 95\% were seeds containing also the seed oil. After dry fractionation about $17 \%$ of the seeds were distributed to fine fraction and the rest - about $83 \%$ - to coarse fraction. Different berry fractions and estimated yields of these fractions are presented in Fig. 1. Estimated values were calculated from averages of berry treatments. Stereo micrographs of cloudberry fractions are presented in Supplementary Material 2.

\subsection{Phenolic composition of the extracts}

Phenolic compounds of fermented and dry fractionated cloudberry samples were analysed by HPLC-DAD and by UPLC-QToFMS/MS using acetone extracts. Altogether 16 phenolic compounds were detected from cloudberry. The UV chromatograms at $280 \mathrm{~nm}$ and $360 \mathrm{~nm}$ showed the presence of ellagitannins, ellagic acid and derivatives, flavonols and hydroxycinnamic acid, ellagitannins and ellagic acid being the most abundant compounds (Table 2). Ellagic acid, ferulic acid, caffeic acid and benzoic acid were detected in comparison with authentic standards. The identification of the remaining peaks was based on the fragmentation data reported in the literature and UV spectrum (Fischer, Carle, \& Kammerer, 2011; Gasperotti, Masuero, Vrhovsek, Guella, \& Mattivi, 2010; God-evac, Tešević, Vajs, Milosavljevic, \& Stankovic, 2009; Kähkönen et al., 2012; McDougall, Martinussen, \& Stewart, 2008; Seeram, Lee, Scheuller, \& Heber, 2006). The MS/MS analysis in negative mode of all ellagitannins found gave a fragment ion at $\mathrm{m} / \mathrm{z}$ 301 representing ellagic acid. The main ellagitannins detected in cloudberry correspond to the dimeric sanguiin $\mathrm{H}-6,[\mathrm{M}-\mathrm{H}]^{-}$at $m / z 1869$, and trimeric lambertianin $C,[\mathrm{M}-2 \mathrm{H}]^{2-}$ at $m / z 1401$ (Kähkönen et al., 2012; McDougall et al., 2008). Other ellagitannins found in cloudberry in low concentration were tentatively identified like isomers of sanguiin $\mathrm{H} 10(\mathrm{~m} / \mathrm{z} 1567)$, casuarictin/ potentillin $(m / z 935)$ and sanguiin $\mathrm{H} 2(\mathrm{~m} / \mathrm{z}$ 1103). The MS/MS study also indicated the presence of ellagic acid-derivatives such

Table 2

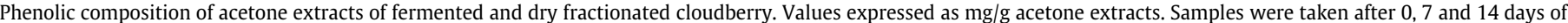
fermentation.

\begin{tabular}{|c|c|c|c|c|c|c|c|}
\hline Peak & & {$[\mathrm{M}-\mathrm{H}]-$} & 0 days & 7 days & 14 days & Dry fraction $\mathrm{D}$ & Dry fraction $\mathrm{E}$ \\
\hline \multicolumn{8}{|c|}{ Ellagitannins } \\
\hline 1 & Sanguiin $\mathrm{H} 10$ & 1565 & 13.46 & 8.60 & 3.58 & 12.79 & 7.69 \\
\hline 2 & Casuarictin/potentillin & 935 & 3.51 & 9.26 & 9.68 & 16.36 & $-^{\mathrm{a}}$ \\
\hline 3 & Casuaricitrin/potentillin & 935 & 5.45 & 8.05 & 0.00 & 0.00 & 0.00 \\
\hline 4 & Lambertianin C & 1401 & 76.57 & 86.93 & 82.93 & 13.13 & 0.00 \\
\hline 5 & Sanguiin H6 & 1868 & 95.44 & 91.89 & 85.90 & 43.82 & 26.48 \\
\hline 6 & Sanguiin $\mathrm{H} 2$ & 1103 & 9.43 & 0.00 & 0.00 & 10.30 & 5.63 \\
\hline 7 & Sanguiin $\mathrm{H} 2$ & 1103 & 4.32 & 3.95 & 3.84 & 13.37 & 9.11 \\
\hline 8 & Casuaricitrin/potentillin & 935 & 6.53 & 6.38 & 7.90 & 6.53 & 1.20 \\
\hline \multirow[t]{2}{*}{9} & Sanguiin $\mathrm{H} 2$ & 1103 & 6.07 & 4.67 & 3.86 & - & 5.40 \\
\hline & Total & & 220.78 & 219.73 & 197.70 & 116.30 & 55.51 \\
\hline \multicolumn{8}{|c|}{ Hydroxycinnamic acids } \\
\hline 10 & Ferulic acid & 163 & 0.83 & 0.00 & 0.00 & 0.00 & 1.59 \\
\hline \multirow[t]{2}{*}{11} & Caffeic acid & 193 & 1.51 & 1.11 & 0.00 & 0.00 & 0.00 \\
\hline & Total & & 2.33 & 1.11 & 0.00 & 0.00 & 1.59 \\
\hline \multicolumn{8}{|c|}{ Flavonols } \\
\hline 12 & Q-3-O-gluc ${ }^{\mathrm{b}}$ & 477 & 4.06 & 5.01 & 5.82 & 0.69 & 1.68 \\
\hline \multirow[t]{2}{*}{13} & Q 3-O- $\left[6^{\prime \prime}\right]^{\mathrm{C}}$ & 607 & 1.07 & 1.21 & 2.34 & 0.41 & 2.73 \\
\hline & Total & & 5.13 & 6.22 & 8.16 & 1.10 & 4.41 \\
\hline \multicolumn{8}{|c|}{ Ellagic acid derivatives } \\
\hline 14 & Ellagic-gly ${ }^{\mathrm{d}}$ & 463 & 0.00 & 12.91 & 15.07 & 7.94 & 20.87 \\
\hline 15 & Ellagic acid & 301 & 144.30 & 262.20 & 508.00 & 122.89 & 119.33 \\
\hline \multirow[t]{2}{*}{16} & Ellagic-acetylpent ${ }^{\mathrm{e}}$ & 475 & 3.66 & 2.74 & 5.05 & 3.54 & 3.65 \\
\hline & Total & & 147.96 & 277.85 & 528.12 & 134.37 & 143.85 \\
\hline
\end{tabular}

Bold values refer to the sum of compounds in each phenolic main groups: ellagitannins etc.

a No detected.

b Q-3-O-gluc. Quercetin-3-O-glucuronide.

c Q 3-O-[6"] Quercetin 3-O-[6"-(3-hydroxy-3methylglutaroyl)- $\beta$-glucoside].

d gly. Glycoside.

e Acetylpent. acetylpentoside. 
Table 3

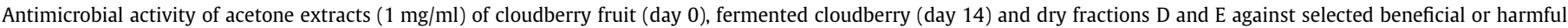
microbial strains.

\begin{tabular}{|c|c|c|c|c|c|c|}
\hline Extract $(1 \mathrm{mg} / \mathrm{ml})$ & S. aureus E-70045 & E. coli E-093121 & P. aeruginosa E-84219 & S. cerevisiae C-00360 & C. albicans C-85161 & L. rhamnosus E-96666 \\
\hline Day 0 & +++++ & ++ & ++ & - & $+1-$ & - \\
\hline Day 14 & +++++ & ++ & ++ & - & $+1-$ & - \\
\hline Dry fraction D & ++++ & $+++^{+}$ & ++ & - & - & nd \\
\hline Dry fraction $\mathrm{E}$ & +++++ & ++ & ++ & - & - & nd \\
\hline
\end{tabular}

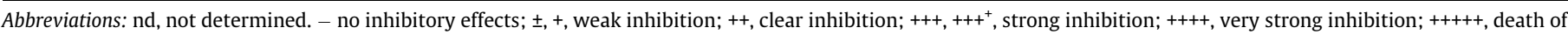
the culture.

as, ellagic acid-glycoside $(\mathrm{m} / \mathrm{z} 463)$ and ellagic acid-acetylpentoside $(\mathrm{m} / \mathrm{z} 475)$, as well as different quercetin derivatives like quercetin3-O-glucuronide $(\mathrm{m} / \mathrm{z} 447)$ and quercetin 3-O-[6"-(3-hydroxy3methylglutaroyl)- $\beta$-glucoside] ( $m / z$ 607).

Accumulation of ellagic acid and simultaneous degradation of ellagitannins was the general trend in cloudberry LAB fermentation. In addition, hydroxycinnamic acids decreased and flavonol derivatives increased.

Before dry fractionation process the juice was separated from the press cake. Distribution of different groups of phenolics between juice and press cake were screened by HPLC. Ellagic acid was equally distributed between juice and press cake, while flavonols and ellagitannins were concentrated in press cake (data not shown).

The phenolic profile of each extract was distinct. Interestingly, phenolic profiles of the seed fine fraction (fraction D) and seed coarse fraction (fraction E) differed remarkably. Seed fine fraction clearly contained more tannins than seed coarse fraction. On the other hand, more flavonols, namely quercetin derivatives, were found in seed coarse fraction. Ellagic acid was equally distributed between the dry fractions.

\subsection{Bioactivities}

\subsubsection{Antimicrobial activity}

Antimicrobial activity was tested in liquid cultures of selected microbial strains using acetone extracts $\left(1 \mathrm{mg} \mathrm{mL}^{-1}\right)$ of cloudberry samples (Table 3). Non-fermented as well as fermented cloudberry was strongly antimicrobial against S. aureus VTT E-70045, and also inhibited growth of pathogenic Gram-negative bacteria $E$. coli VTT E-093121 and $P$. aeruginosa VTT E-84219. These results are well in line with our earlier studies (Nohynek et al., 2006; PuupponenPimiä et al., 2005). Fermentation did not alter antimicrobial activity. The growth of pathogen yeast strain C. albicans VTT C-85161 was slightly decelerated with the cloudberry extracts. Extracts did not have an effect on growth of probiotic strain L. rhamnosus VTT E-96666 and spoilage yeast S. cerevisiae VTT C-00360. Dry fractions of fermented cloudberry showed variable antimicrobial activities against microbial species in this study. S. aureus was the most sensitive to all the acetone extracts of the dry fractions. In addition, seed fine and coarse fractions of fermented cloudberry were active against uropathogenic $E$. coli and $P$. aeruginosa. S. cerevisiae and $C$. albicans were not sensitive against any of the dry fractions tested. Interestingly, the extract from seed fine fraction showed stronger inhibition on the growth of uropathogenic E. coli than other cloudberry extracts. Concentrations of casuarictin/potentillin (peak 2, Table 2) and sanguiin H2 (peak 7, Table 2) isomers were higher in this extract compared to any other tested extract. This might be one reason for increased antimicrobial activity. Phenolics may also act in synergistic way, and thus specific combination of phenolics in seed fine fraction might have been most effective against E. coli. Antimicrobial activity may also be dependent on other compounds as phenolics. Berry seed oil is also well known for its antimicrobial activity. Distribution of seed oil components between seed dry fractions was not studied. Vattem, Lin, Labbe, and Shetty $(2004,2005)$ have used solid state bioprocessing to enhance health benefits of berries through phenolic antioxidant enrichment. In their experiments cranberry pomace was bioprocessed by food-grade fungus Rhizopus oligosporus or Lentinus edode. This bioprocessing increased antioxidant activity and antimicrobial activity against important foodborne pathogens, such as Listeria monocytogenes, Vibrio parahaemolyticus, and E. coli 0157:H7.

\subsubsection{Anti-adhesion activity}

Acetone extracts of cloudberry fermentations and dry fractions were analysed for the minimum inhibitory concentration (MIC) required to inhibit $\mathrm{HA}$ of the recombinant strain E. coli HB101 (pPIL291-15), which expresses $P$ fimbriae as the sole fimbria type. E. coli HB101 (pPIL291-15) efficiently agglutinated human erythrocytes in the absence of inhibitor, whereas the well-characterised non-fimbriated laboratory strain E. coli HB101, used as a control in the study, did not agglutinate the erythrocytes. As shown in Fig. 2, unfermented extract (day 0 ) inhibited HA of P-fimbriated E. coli at an average of $7 \mu \mathrm{g}$ extract $/ \mathrm{mL}$. The extract at fermentation

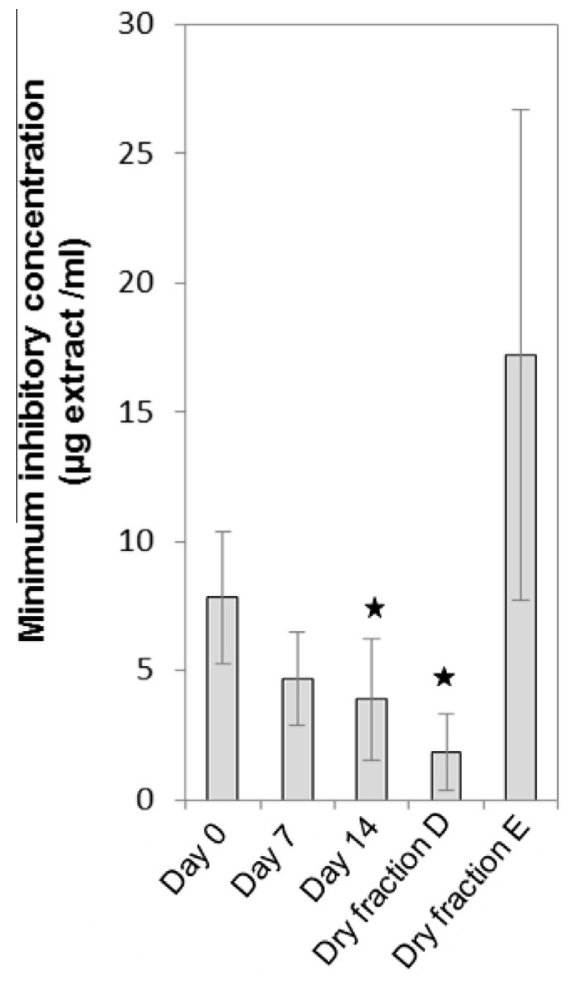

\section{Acetone extract}

Fig. 2. Effect of cloudberry phenolic extracts on bacterial adhesion. Minimum concentrations of acetone extracts $(\mu \mathrm{g} / \mathrm{mL})$ of fermented cloudberry (days $0,7,14$ ), and seeds dry fractions (D, fine fraction; E, coarse fraction) required for inhibition of P-fimbriated $E$. coli to human erythrocytes are shown. The results represent averages and standard deviations of four independent experiments. Significant difference between a sample extract and the extract of day 0 fermentation, as calculated by Student's $t$-test, is indicated with an asterisk ( $\left.{ }^{*} p<0.05\right)$. 
midpoint (day 7) inhibited the HA at an average of $5 \mu \mathrm{g}$ extract $/ \mathrm{mL}$, but the difference to the control was not statistically significant. The extract at the end of fermentation (day 14) showed a statistically significantly lower MIC of $3.9 \mu \mathrm{g}$ extract $/ \mathrm{mL}$ compared to the unfermented extract. The extract of the coarse seed fraction (fraction E) showed low inhibitory capacity; the MIC was on average $17 \mu$ g extract $/ \mathrm{mL}$. Interestingly, the extract of the fine seed fraction (fraction D) was the most potent inhibitor of E. coli HA, showing an MIC of only $1.9 \mu \mathrm{g}$ extract $/ \mathrm{ml}$, which statistically is significantly different from the non-fermented control extract.

The concentrations (as $\mathrm{mg} / \mathrm{g}$ extract) of individual phenolic compounds in the acetone extracts were determined (Table 2) and the results were used in combination with the obtained MIC values ( $\mu$ g extract/mL, Fig. 2) to determine the concentration of ellagitannin, hydroxycinnamic acid, flavonols and ellagic acid and derivatives at the MIC of the various acetone extracts (see Supplementary Material 3). The results showed that the most potent inhibitory acetone extract, the fine seed fraction $\mathrm{D}$, contained the lowest concentrations of phenolic compounds. The

A

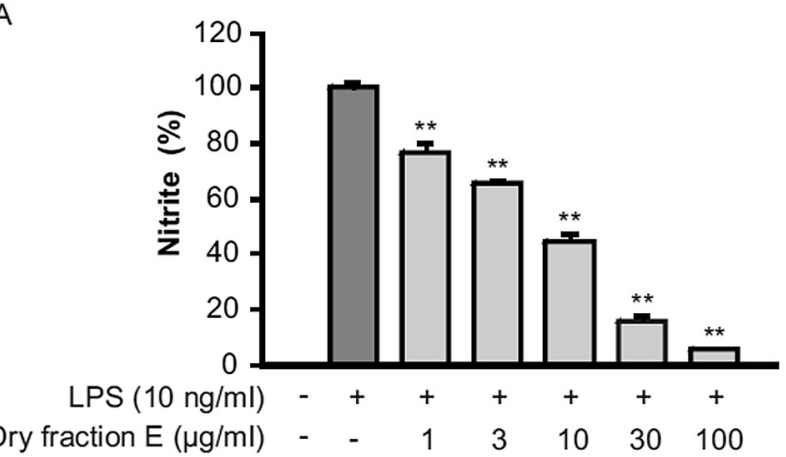

B

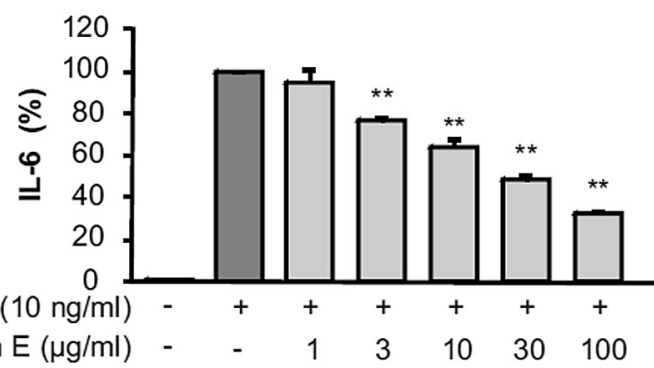

C

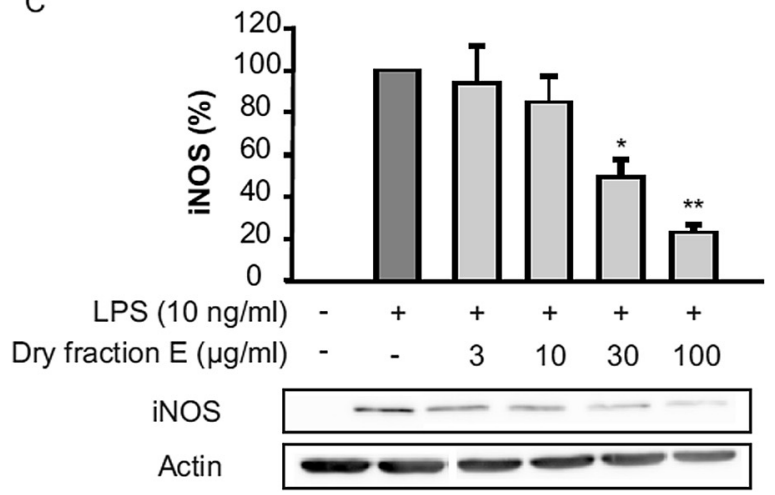

Fig. 3. Effects of the coarse dry fraction (fraction $E$ ) on nitric oxide (A) and interleukin 6 (IL-6; B) production and on inducible nitric oxide synthase (iNOS) expression in activated macrophages. Nitric oxide production (A) was determined by measuring the accumulation of its stable metabolite nitrite into the culture medium. Values are mean $\pm \mathrm{SEM}, n=4 ;{ }^{*} p<0.05,{ }^{* *} p<0.01$. major components in the fine seed fraction D at MIC (ellagic acid and derivatives, ellagitannin, benzoic acid) were present at much higher concentrations in the extracts of lower inhibitory potential. The results indicate that the inhibitory compound was not one of the phenolic compounds. Due to the importance of $\mathrm{P}$ fimbriamediated adhesion in the virulence of uropathogenic $E$. coli, several anti-adhesive approaches have been reported. These include inhibition of P-fimbrial binding to target cells using synthetic derivatives

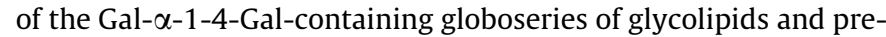
vention of $P$ fimbria assembly on the bacterial surface using rationally designed synthetic inhibitors of fimbria assembly (reviewed by Ruer, Pinotsis, Steadman, Waksman, and Remaut (2015)).

\subsubsection{Anti-inflammatory activity}

The anti-inflammatory properties of cloudberry acetone extracts were tested in macrophages activated by exposure to bacterial endotoxin LPS. Under these conditions, the cells produce proinflammatory factors, such as NO, IL-6 and iNOS, which were measured in the present study. The effects of the cloudberry acetone extracts on the inflammatory factors were investigated by adding the extracts in five concentrations $(1,3,10,30$, and $100 \mu \mathrm{g} / \mathrm{mL}$ ) into the cultures of activated macrophages. The extracts did not show cytotoxic effects but some of them had interesting anti-inflammatory properties (see Supplementary Material 4).

Unfermented (day 0) extract at higher concentrations had a minor anti-inflammatory effect on NO and IL-6 production. Interestingly, the fermented (day 14) extract showed somewhat improved anti-inflammatory activity as compared to the unfermented (day 0) extract, suggesting that the fermentation process produced/enriched anti-inflammatory compounds in the berry material.

When investigating the anti-inflammatory effects of the extracts of dry fractions (fractions D and E) the fine fraction (fraction D) showed only minor anti-inflammatory activity. Intriguingly, the extract of the coarse dry fraction (fraction E) had the most potent anti-inflammatory properties among the tested extracts. It reduced NO and IL-6 production and iNOS expression in a dose-dependent manner and produced statistically significant effects already at concentrations of $1-3 \mu \mathrm{g} / \mathrm{ml}$ (Fig. 3). These results suggest that the anti-inflammatory compounds produced during the fermentation process are concentrated in the coarse dry fraction (fraction E).

As described above, the phenolic profiles of the four tested acetone extracts were distinct. Also, the phenolic profiles of the two dry fractions were different and quercetin derivatives were shown to concentrate in the coarse dry fraction E. Quercetin has been shown to possess anti-inflammatory properties in activated macrophages (Endalea et al., 2013; Hämäläinen, Nieminen, Vuorela, Heinonen, \& Moilanen, 2007; Jung \& Sung, 2004), as well as in carrageenan-induced inflammation in rats (Morikawa et al., 2003). In activated macrophages the effects of quercetin on transcription factors NF-kappaB and STAT1 have been proposed to explain, at least partly, the inhibitory effects of quercetin on NO production and iNOS expression (Hämäläinen et al., 2007).

\section{Conclusion}

Phenolic composition and bioactivity of cloudberry can be remarkably modified by bioprocessing. In addition, highly bioactive fractions can be produced by dry fractionation of the press cake. During fermentation ellagitannins were degraded and simultaneously ellagic acid was accumulated. Phenolics were differentially distributed in seed coarse and fine fractions after dry fractionation process. Seed fine fraction contained clearly more 
tannins than seed coarse fraction. On the other hand, more quercetin derivatives were found in seed coarse fraction. Ellagic acid was equally distributed between the dry fractions.

Fermentation did not increase antimicrobial activity of cloudberry, but interestingly dry fractionation of the press cake did, as seed fine fraction had some increased antimicrobial activity against uropathogenic E. coli.

Due to the significance of $\mathrm{P}$ fimbria-mediated adhesion in the virulence of uropathogenic $E$. coli, various anti-adhesive approaches have been reported. However, the reported P-fimbria inhibitors are synthetic and their synthesis complicated or expensive. Our results clearly showed that naturally occurring cloudberry phenolics have potential as competitive inhibitors of $E$. coli adhesion. Fermentation had a minor, but statistically significant effect on the anti-adhesive capacity of the cloudberry, which was seen as inhibition of P fimbria-mediated HA of E. coli. The extracts of the seed fractions differed markedly, as the fine seed fraction had a significantly higher inhibitory effect on $E$. coli adhesion than the coarse seed fraction.

Our results also showed that fermentation process enriched or produced anti-inflammatory compounds of the cloudberry material. Interestingly, the seed coarse fraction was shown to be very potent as it significantly reduced NO and IL-6 production and iNOS expression even at low concentration.

It was not possible to identify phenolic compounds from the berry material that caused inhibition of growth and fimbriamediated adhesion of $E$. coli bacteria or reduced production of inflammatory biomarkers. These components may not be identifiable in the current study or they may be non-phenolic compounds. Synergistic effects of specific phenolics are also possible. In general cloudberry proved to be a very potent source for bioactive compounds. This is very important, because berries can be easily added to the normal daily diet, and thus patients and other needy persons would get these health benefits in a regular manner. The results provide interesting suggestions for future research and applications: the active compounds present in the seeds should be identified and further evaluated in pharma or health food applications.

\section{Conflict of interest}

The authors declare no conflicts of interest.

\section{Acknowledgements}

Tekes- the Finnish Funding Agency for Technology and Innovation (BERRYFERMENTATION project (No. 40437/08) during the years 2009-2012) and Medical Research Fund of Tampere University Hospital are acknowledged for financial support. We wish to thank Airi Hyrkäs, Liisa Änäkäinen, Pirkko Nousiainen, Heidi Eriksson, Merja Salmijärvi, Ulla Österlund, Salla Hietakangas, Mirva Jär velä-Stölting, Meiju Kukkonen, Maiju Laaksonen and Petra Miikkulainen for expert technical assistance and Päivi Vahala and Heli Määttä for skilful secretarial help.

\section{Appendix A. Supplementary data}

Supplementary data associated with this article can be found, in the online version, at http://dx.doi.org/10.1016/j.foodchem.2015. 11.061.

\section{References}

Aaby, K., Ekeberg, D., \& Skrede, G. (2007). Characterization of phenolic compounds in strawberry (Fragaria $\mathrm{x}$ ananassa) fruits by different HPLC detectors and contribution of individual compounds to total antioxidant capacity. Journal of Agricultural and Food Chemistry, 55, 4395-4406.
Boyer, H. W., \& Roulland-Dussoix, D. (1969). A complementation analysis of the restriction and modification of DNA in Escherichia coli. Journal of Molecular Biology, 41, 459-472.

Endalea, M., Parka, S.-C., Kimb, S., Kimc, S.-H., Yangd, Y., Chod, J. Y., \& Rheea, M. H. (2013). Quercetin disrupts tyrosine-phosphorylated phosphatidylinositol 3kinase and myeloid differentiation factor-88 association, and inhibits MAPK/AP1 and IKK/NF-kB-induced inflammatory mediators production in RAW 264.7 cells. Immunobiology, 218, 1452-1467.

Fischer, U. A., Carle, R., \& Kammerer, D. R. (2011). Identification and quantification of phenolic compounds from pomegranate (Punica granatum L.) peel, mesocarp, aril and differently produced juices by HPLC-DAD-ESI/MSn. Food Chemistry, 127, $807-821$.

Gasperotti, M., Masuero, D., Vrhovsek, U., Guella, G., \& Mattivi, F. (2010). Profiling and accurate quantification of rubus ellagitannins and ellagic acid conjugates using direct UPLC-Q-TOF HDMS and HPLC-DAD analysis. Journal of Agricultural and Food Chemistry, 58, 4602-4616.

God-evac, D., Tešević, V., Vajs, V., Milosavljevic, S., \& Stankovic, M. (2009). Antioxidant properties of raspberry seed extracts on micronucleus distribution in peripheral blood lymphocytes. Food and Chemical Toxicology, 47, 2853-2859.

Green, L. C., Wagner, D. A., Glogowski, J., Skipper, P. L., Wishnok, J. S., et al. (1982). Analysis of nitrate, nitrite, and [15N]nitrate in biological fluids. Analytical Biochemistry, 126, 131-138.

Hämäläinen, M., Nieminen, R., Vuorela, P., Heinonen, M., \& Moilanen, E. (2007). Anti-inflammatory effects of flavonoids: genistein, kaempferol, quercetin, and daidzein inhibit STAT-1 and NF-kappaB activations, whereas flavone, isorhamnetin, naringenin, and pelargonidin inhibit only NF-kappaB activation along with their inhibitory effect on iNOS expression and NO production in activated macrophages. Mediators of Inflammation, 2007, 45673.

Jung, W.-J., \& Sung, M.-K. (2004). Effects of major dietary antioxidants on inflammatory markers of RAW 264.7 macrophages. BioFactors, 21, 113-117.

Kähkönen, M. P., Hopia, A. I., \& Heinonen, M. (2001). Berry phenolics and their antioxidant activity. Journal of Agricultureal and Food Chemistry, 49, 4076-4082.

Kähkönen, M., Kylli, P., Ollilainen, V., Salminen, J.-P., \& Heinonen, M. (2012). Antioxidant activity of isolated ellagitannins from red raspberries and cloudberries. Journal of Agricultural and Food Chemistry, 60, 1167-1174.

Katina, K., Laitila, A., Juvonen, R., Liukkonen, K., Kariluoto, S., Piironen, V., Poutanen, K. (2007). Bran fermentation as a means to enhance technological properties and bioactivity of rye. Food Microbiology, 24, 175-186.

Kylli, P., Nohynek, L., Puupponen-Pimiä, R., Westerlund-Wikström, B., Mcdougall, G., Stewart, D., \& Heinonen, M. (2010). Rowanberry phenolics: Compositional analysis and bioactivities. Journal of Agricultural and Food Chemistry, 58, 11985-11992.

Larrosa, M., García-Conesa, M. T., Espín, J. C., \& Tomás-Barberán, F. A. (2010). Ellagitannins, ellagic acid and vascular health. Molecular Aspects of Medicine, 31, 513-539.

Leppänen, T., Jalonen, U., Korhonen, R., Tuominen, R. K., \& Moilanen, E. (2010). Inhibition of protein kinase Cdelta reduces tristetraprolin expression by destabilizing its mRNA in activated macrophages. European Journal of Pharmacology, 628, 220-225.

Määttä-Riihinen, K., Kamal-Eldin, A., \& Törrönen, R. (2004). Identification and quantification of phenolic compounds in berries of Fragaria and Rubus species (Family Rosaceae). Journal of Agricultural and Food Chemistry, 52, 6178-6187.

McDougall, G., Martinussen, I., \& Stewart, D. (2008). Towards fruitful metabolomics: High throughput analyses of polyphenol composition in berries using direct infusion mass spectrometry. Journal of Chromatography, 871, 362-369.

Morikawa, K., Nonaka, M., Narahara, M., Torii, I., Kawaguchi, K., Yoshikawa, T., ... Morikawa, S. (2003). Inhibitory effect of quercetin on carrageenan-induced inflammation in rats. Life Sciences, 74, 709-721.

Nohynek, L. J., Alakomi, H.-L., Kähkönen, M. P., Heinonen, M., Helander, I. M., Oksman-Caldentey, K.-M., \& Puupponen-Pimiä, R. H. (2006). Berry phenolics: antimicrobial properties and mechanisms of action against severe human pathogens. Nutrition and Cancer, 54, 18-32.

Puupponen-Pimiä, R., Nohynek, L., Alakomi, H.-L., \& Oksman-Caldentey, K.-M. (2005). Bioactive berry compounds - novel tools against human pathogens. Applied Microbiology and Biotechnology, 67, 8-18.

Puupponen-Pimiä, R., Nohynek, L., Ammann, S., Oksman-Caldentey, K.-M., \& Buchert, J. (2008). Enzyme-assisted processing increases antimicrobial and antioxidant activity of bilberry. Journal of Agricultural and Food Chemistry, 56, 681-688.

Puupponen-Pimiä, R., Seppänen-Laakso, T., Maukonen, J., Törrönen, R., Kolehmainen, M., Kankainen, M., ... Oksman-Caldentey, K.-M. (2013). Effects of ellagitannin rich berries on blood lipids, gut microbiota and urolithin production in human subjects with symptoms of metabolic syndrome. Molecular Nutrition and Food Research, 57, 2258-2263.

Rojanathammanee, L., Puig, K. L., \& Combs, C. K. (2013). Pomegranate polyphenols and extract inhibit nuclear factor of activated T-cell activity and microglial activation in vitro and in a transgenic mouse model of Alzheimer disease. Journal of Nutrition, 143, 597-605.

Ruer, S., Pinotsis, N., Steadman, D., Waksman, G., \& Remaut, H. (2015). Virulencetargeted antibacterials: Concept, promise, and susceptibility to resistance mechanisms. Chemical Biology \&' Drug Design, 86, 379-399.

Sangiovanni, E., Vrhovsek, U., Rossoni, G., Colombo, E., Brunelli, C., Brembati, C., ... Dell'Agli, M. (2013). Ellagitannins from Rubus berries for the control of gastric inflammation: in vitro and in vivo studies. PLoS One, 8, e71762. 
Seeram, N., Lee, R. L. H. S., Scheuller, S., \& Heber, D. (2006). Identification of phenolic compounds in strawberries by liquid chromatography electrospray ionization mass spectroscopy. Food Chemistry, 97, 1-11.

Sheng, J., Baldeck, J. D., Nguyen, P. T., Quivey, R. G., Jr., \& Marquis, R. E. (2010). Alkali production associated with malolactic fermentation by oral streptococci and protection against acid, oxidative, or starvation damage. Canadian Journal of Microbiology, 56, 539-547.

Signoretto, C., Canepari, P., Stauder, M., Vezzulli, L., \& Pruzzo, C. (2012). Functiona foods and strategies contrasting bacterial adhesion. Current Opinion in Biotechnology, 23, 160-167.

van Die, I., van Megen, I., Hoekstra, W., \& Bergmans, H. (1984). Molecular organisation of the genes involved in the production of $\mathrm{F}_{(2)}$ fimbriae, causing mannose-resistant haemagglutination, of a uropathogenic Escherichia coli 06: K2:H1:F7 strain. Molecular Genomics and Genetics, 194, 528-533.
Vattem, D. A., Lin, Y.-T., Labbe, R. G., \& Shetty, K. (2004). Antimicrobial activity against select food-borne pathogens by phenolic antioxidants enriched in cranberry pomace by solid-state bioprocessing using the food grade fungus Rhizopus oligosporus. Process Biochemistry, 39, 1939-1946.

Vattem, D. A, Lin, Y.T. Labbe, R. G. \& Shetty, K. (2005). Phenolic antioxidant mobilization in cranberry pomace by solid-state bioprocessing using food grade fungus Lentinus edodes and effect on antimicrobial activity against select food borne pathogens. Innovative Food Science and Emerging Technologies, 5, 81-91.

Viljanen, K., Heiniö, R.-L., Juvonen, R., Kössö, T., \& Puupponen-Pimiä, R. (2014) Relation of sensory perception with chemical composition of bioprocessed lingonberry. Food Chemistry, 157, 148-156. 